\title{
Circuit
}

Musiques contemporaines

\section{Bruit, son, silence : le bruit - prospective négative et prospective positive}

\section{Gilles Tremblay}

Volume 5, numéro 1, 1994

Gilles Tremblay : réflexions

URI : https://id.erudit.org/iderudit/902087ar

DOI : https://doi.org/10.7202/902087ar

Aller au sommaire du numéro

Éditeur(s)

Les Presses de l'Université de Montréal

ISSN

1183-1693 (imprimé)

1488-9692 (numérique)

Découvrir la revue

Citer cet article

Tremblay, G. (1994). Bruit, son, silence : le bruit - prospective négative et prospective positive. Circuit, 5(1), 29-36. https://doi.org/10.7202/902087ar d'utilisation que vous pouvez consulter en ligne.

https://apropos.erudit.org/fr/usagers/politique-dutilisation/ 


\section{BRUIT, SON, SILENCE}

\section{Le bruit - prospective négative et prospective positive}

Paru dans l'ouvrage collectif, Le Bruit, Centre d'études prospectives du Québec, P.U.M., 1970.

Dissipons d'abord un malentendu.

«Ce qui est à éviter dans un contexte donné », telle est la définition commune du bruit.

Le théoricien de la musique (suivant et interprétant une démarche parallèle à celle de l'acousticien) affirme de son côté : «Le son musical se distingue du bruit en ce qu'on peut en mesurer exactement la hauteur, tandis qu'on ne peut apprécier la valeur musicale d'un bruit " "').

Déjà, le sens s'est rétréci. On appelle bruit uniquement les phénomènes sonores dont « on ne peut mesurer la hauteur "; implicitement, l'affirmation du théoricien sous-entend que le bruit (phénomène sonore rebelle à la mesure à cause de ses vibrations irrégulières) ne peut avoir de "valeur musicale " n'étant réservé qu'aux sons dont on peut apprécier la hauteur là vibrations régulières). On voit ici comment la notion large (de la définition commune) revient subrepticement à la surface provoquant un glissement: tout ce qui n'a pas de hauteur définie, le bruit, est à éviter. Nous sommes au cœur du malentendu issu de la superposition de deux notions complètement différentes, couvertes par un seul mot, «bruit». Sous cette cache unique, elles ont de malignes tendances à se substituer l'une à l'autre, piège rendant souvent la réflexion sinon malaisée du moins vulnérable, comme nous l'avons expérimenté à plusieurs reprises. Un exemple nous aidera à mieux le discerner.

Supposons un auditeur à qui l'on fait entendre un son très « musical » à hauteur définie et très pur (comme peut l'être par exemple un son de flûte ou mieux, un son sinusoïdal d'origine électro-acoustique), disons un mi à 600 vibrations par seconde, dans une nuance qui ne sera même pas forte mais
(1) A. Danhauser: Théorie de la musique, Paris, H. Lemoine \& Cie, 1929, p. 119 , note $\mathrm{A}$. 
moyenne. On peut être assuré que cette personne, au bout d'un certain laps de temps, selon son degré de résistance et de patience, trouvera ce son très peu désirable et à éviter, véritable scie pour les oreilles. Pourtant, il ne s'agissait nullement d'un «bruit» selon notre théoricien, mais bien d'un "son musical ».

Par contre, le même individu aurait sans doute à durée égale apprécié davantage certains bruits: celui d'une fontaine, de la pluie, d'un feu qui crépite ou des vagues de la mer, par exemple.

Poussons plus loin l'expérience et nous découvrirons qu'un même phénomène sonore peut avoir des sens diamétralement opposés selon le point de vue où l'on se place. Pour le musicien que je suis, la longue clameur céleste des avions à réaction est un vaste signe poétique en sa courbe de plénitude (calligraphie et «calliphonie» à la fois). Aurais-je la même impression si, comme des milliers de personnes, $j$ 'habitais dans le voisinage d'un aéroport? Pourtant, ne s'agit-il pas d'une seule et unique source sonore?

La différence de direction (et de dimension) des deux écoutes se situe sans doute dans la qualité du silence de chacune. J'anticipe en nommant ce mot, mais c'est à dessein que je le fais, car il est trop intimement lié au sujet pour qu'on ne l'y associe pas dès maintenant.

Ces trois exemples (et surtout le dernier), on en a l'intuition, nous donnent le pressentiment et nous mettent déjà sur la piste de ce que nous appelons prospective négative et prospective positive. Le double piège est repéré, désamorçons-le complètement en précisant le sens employé dans chacun des cas.

Dans la prospective négative le mot bruit désignera exclusivement «Tout son, quelles que soient son origine et sa nature, qu'on s'efforce, dans des conditions données, d'éviter ${ }^{(2)}$.

Dans la perspective positive, il sera question de la perception acceptée et désirée de tous les phénomènes sonores existant depuis l'événement le plus familier jusqu'au plus inouĩ et au plus étranger.

\section{Prospective négative}

Pour communiquer, parler à quelqu'un par exemple, il faut, près de celui qui reçoit ou écoute, un minimum de silence. Chacun peut expérimenter la situation où une foule réunie dans une même enceinte est formée de petits
(2) Voir «Bruit * dans l'Encyclopédie de la musique, Fasquelle, 1958, vol. I. 
groupes qui discutent entre eux. Très rapidement, un mécanisme de crescendo se met en branle, chaque interlocuteur parle plus fort que son voisin et aussi plus haut. Dans ce processus de surenchère, au bout d'un certain temps tout le monde crie à s'égosiller. Et puis chacun, assourdi et hurlant, ne voit plus que des centaines de lèvres muettes remuant avec volubilité d'innombrables phonèmes que personne n'entend, un peu comme au cinéma muet. Sans doute pense-til à ce moment, celui qui voit cela et tonitrue tout à la fois, qu'il pourrait tout aussi bien aboyer ou braire ${ }^{(3)}$.

Et peut-être est-il tenté de le faire et le fait-il ? (Réaction beaucoup plus saine et sage qu'on serait tenté de le croire). À moins que, au moment de succomber à la tentation, il en soit empêché par épuisement des cordes vocales /ce qui serait également un début de sagessel.

Projeter ce processus de surenchère dans d'autres domaines afin d'en discerner l'amorce dans le monde où nous vivons. En déduire le signe.

Distinguons auparavant les nuances de situation. D'abord une première différence de base: notre foule de tout à l'heure pouvait et devait s'arrêter ne serait-ce que par épuisement, alors que le crescendo et la masse d'information actuels (je pense aux sources mécaniques et électroniques) ont la possibilité de poursuivre leur prolifération sans fatigue. II n'y a aucun motif d'épuisement pour un haut-parleur. S'il y a usure, on ne peut la mettre en parallèle avec l'épuisement. Seconde différence de base: les sources sonores sont infiniment moins contrôlées par ceux qui les reçoivent et nullement dans certains cas. Prenez par exemple celui des musiques d'ambiance des lieux publics (magasins, restaurants, moyens de transport, etc.) sans parler du mouvement unilatéral de la radio-télévision, où cela va de soi, il est malaisé de contredire un interlocuteur, ou simplement de témoigner de sa réaction. Troisième différence enfin: dans l'enceinte aux groupes multiples, celui qui le désirait avait toujours la possibilité de sortir, alors que dans le monde que nous imaginons, cette possibilité n'existera plus, tout étant pollué, nous serons à « huis-clos » sur notre planète. - On peut d'ailleurs diagnostiquer dès maintenant par une sorte d'écho anticipé du futur l'indice révélateur de la saturation-pollution du lieu lorsque l'espace-silence est présenté comme un luxe: I'aspect achat-évasion de certains prospectus d'agences touristiques; la cure (de silence) Vittel; l'absence de musique douce uniquement en première classe dans les trains; l'insonorisation des appartements de grand luxe. Autre indice, la disparition progressive de certaines zones que l'on aurait pu croire intangibles: la musique « d'ambiance » des espaces verts urbains là Montréal, la zone du Lac des
(3) L'étymologie ne nous apprend-elle pas que bruire vient du latin vulgaire brugere, croisement entre le latin rugire, rugir, et bragere, braire? 
Castors, sur le Mont-Royal); de la plupart des pentes de ski des Laurentides. Sans parler de cet inénarrable nomade qu'est un transistor dans la nature.

Pour revenir à notre projection, ces différences, loin de s'affaiblir, accentuent au contraire l'aspect emballement d'un processus de moins en moins contrôlé. Tournoiement d'aiguilles folles. En déduire le signe, disons-nous? ॥ se révèle en paradoxe : la multiplication des informations conduit par saturation, à l'absence d'information (signe de l'insignifiant), à l'impossibilité donc de communiquer, en une assourdissante entropie des messages, avec les conséquences ultimes que cela entrainerait: paralysie, mort?

Si nous avons atteint d'un bond le seuil de l'absurde, ce n'est pas, on l'aura deviné, par un pessimisme aussi stérile que vain, mais plutôt pour mieux tendre le possible par l'imaginaire. La réalité pressentie n'est point de tout repos, certes, mais elle ne déclenchera pas pour autant un réflexe d'autruche qui en la masquant, masquerait en même temps par sa démission toute confiance ${ }^{(4)}$.

Plusieurs enquêtes ont déjà été menées dans le monde sur ce sujet. Notre démarche n'étant point celle de l'enquête (ce qui ferait double emploi) mais plutôt celle de la réflexion, c'est se placer au cœur du propos et non le déborder que de réfléchir sur l'enquête.

Comment appréhender les secteurs les plus atteints? Deux coupes s'imposent: une coupe espace, une coupe temps. Elles s'éclaireront l'une l'autre.

Dans la coupe espace, on s'appliquera à situer où est le bruit. $\AA$ titre d'exemple, on analysera les zones urbaines, les lieux de travail, les transports en commun, les bruits industriels (matériau fort riche et proliférant), le bruit dans l'architecture (notamment dans I'habitation), la «musique douce" dans les magasins, les moyens de transport, les restaurants, les ascenseurs, les couloirs, les banques, la rue, etc...

Cette recherche ne consistera pas uniquement à enregistrer des résultats mais aussi à les comprendre en remontant toujours à la source. (Englobons dans «source " tant, s'il y a lieu, l'enceinte acoustique que le générateur sonore).

Sauf pour la «musique douce» (qui, à cause du glissement de sens mentionné plus haut mais ici à rebours, est rarement considérée comme un bruit par les enquêteurs) c'est en général la coupe espace qui est la mieux connue par les spécialistes. Non que la coupe temps soit totalement négligée mais, bien que cela étonne, elle attire ordinairement beaucoup moins l'attention.
(4) Nous aurions affaire ici à un bruit au second degré, ce bruit psychologique est un masque de masque. 
Dans la coupe temps, on s'attachera à savoir quand se produit le bruit, à quel moment. Notamment dans le temps de l'homme. Cette chronologie du bruit ne sera donc pas uniquement quantitative (temps de l'horloge) mais aussi qualitative là quel moment dans le temps de l'homme ? Par exemple, un marteau compresseur n'aura pas la même valeur qualitative à quatre heures du matin qu'à quatre heures de l'après-midil. On pourra étudier les bruits aux différents moments de la journée: sommeil, repas, travail, jeux, études, échanges, etc.; de l'année, selon les saisons; et enfin de la vie en ses différents âges depuis la conception jusqu'à la mort. Le bruit et la mort, ce sujet seul pourrait donner lieu à plusieurs ouvrages!

À la suite du temps de l'homme, on imagine les perspectives qu'ouvre l'étude de l'incidence d'autres temps: le temps biologique en particulier, pour n'en citer qu'un seul(5).

Enfin, pour déterminer les aspects les plus néfastes, on distinguera deux espèces de bruits qui serviront également de critères dans l'orientation de la recherche.

a) Tous ceux qui, par leur morphologie, peuvent occasionner un traumatisme physiologique. Exemples:

1. par l'intensité (mesurable en décibels: lésion de l'appareil auditif);

2. par la fréquence: à intensité égale l'aigu est plus dangereux que le grave;

3. par la longueur de la durée combinée avec l'homogénéité : la fatigue. La meilleure illustration est l'impression de soulagement que l'on peut ressentir au moment de l'arrêt d'une vibration ultra-grave (soufflerie, moteur) que l'on ne percevait même pas consciemment. Son arrêt donne l'impression d'être libéré d'un long effort ;

4. par l'isochronie qui provoque également une fatigue parente de celle de l'homogénéité, et analogue au supplice chinois de la goutte d'eau;

5. par l'inattendu (explosions, «bang " d'avions supersoniques); il ajoute un choc nerveux à la lésion de l'intensité, etc.

b) Tous ceux qui, traités globalement (et non à cause d'une forme particulière), provoquent un phénomène de dispersion d'anti-recueillement, de brouillage formidable. Ils comprennent non seulement les bruits sonores (ce qui semble être un pléonasme), mais aussi les bruits silencieux, ceux de la pensée, ceux qui sont provoqués par une densité d'événements trop grande pour la capacité d'absorption de l'individu (nous touchons ici encore l'idée de saturation «insignification» dont nous avons parlé
(5) On aura compris que cette méthode par coupe espace et temps s'applique aussi bien à une prospective positive qu'à une prospective négative. 
plus haut et qui s'impose comme un des aspects les plus prospectifs du bruit).

Ces bruits vident l'être, le déchargent de ses forces, le rendent finalement insensé. Ce sont peutêtre en fait les plus dangereux et pervers: on ne peut les mesurer en décibels. Pourrait-on jauger en mesures une attaque aussi subtile, aussi spirituelle dans son offensive ?(o) $^{(0)}$

Dans ce domaine, un des secteurs les plus « développés» et dont la prolifération est d'une prospérité sans précédent, alimenté et appuyé par toute la force d'uniformisation que représentent les grandes sociétés de masse là l'Ouest comme à l'Est, bien qu'elles utilisent ce moyen pour des fins différentes), c'est le conditionnement sonore; les musiques douces que l'on trouve à peu près partout en Occident, le slogan incantatoire, politique ou publicitaire, fils du lavage de cerveau via les réflexes conditionnés, bien commun des empires rivaux: deux aliénations. Si la première (musique douce) est proche parente du second (slogan), elle nous apparaît infiniment plus nocive. Le second en sa brutalité a plus de chance de choquer et de provoquer une réaction, alors que la première en sa non-agressivité même est plus pénétrante et insidieuse. À titre d'exemple citons cette publicité musak en 1969: \& Ils (les clients) achètent plus, et pas seulement ce dont ils ont absolument besoin.» «[...] Elle (la musique de Muzak) se distingue par la caractéristique particulière de ne jamais exiger la participation consciente de son auditoire.» Par contre: «[...] La faculté de pouvoir ainsi régler le niveau des effets stimulants sur l'état d'âme permet à Muzak de présenter des programmes spéciaux appropriés à un moment donné, en un lieu donné [...] ».

Ainsi, à la participation consciente libre est substituée une participation inconsciente imposée et conditionnante, l'auditeur-acheteur-ou-travailleur n'ayant aucune action sur la source sono-commerciale. Atteinte à la liberté non seulement parce qu'elle baigne de ses flots le « consommateur ", qu'il le veuille ou non, mais surtout parce qu'elle conditionne le psychisme même, en l'envahissant et en l'imbibant. Suprême hypocrisie, cette violation peut s'effectuer sous le couvert même de la liberté, celle de la libre entreprise. Quant aux arguments de vente des producteurs de "musique douce", ce sont ceux de la consommation: les statistiques ou mieux une période probatoire d'essai prouvent au commerçant que les ventes augmentent quand on «met » de la musique. Argument de «valeur » et décisif, il scelle la complicité en faisant bon marché du bien commun.
(6) On peut juger de la carence d'une enquête faite exclusivement au \& décibelmètre ", surtout lorsqu'elle est présentée comme une enquête sur le bruit alors qu'elle ne rend comple que de l'intensité, se cantonnant ainsi d̀ une seule partie de l'aspect morphologique. 
La musique douce se doit d'être ordinaire, optimiste (pas trop) pas trop forte ni trop faible, lisse, sans aspérité, sans trop de modulations, ni trop lente ni trop rapide, en un mot très homogénéisée. Sa loi structurale est donc la fade moyenne, correspondant à la banalité d'un conforme rose. Tout élément d'imprévisible en étant de nature exclu, on peut aisément confier sa fabrication à un ordinateur sourd mais « conditionné » par un programme adéquat.

Réaction recueillie : «Quand j'entends de la musique en faisant mon marché, je ne puis plus penser, alors j'achète n'importe quoi, même des choses dont je n'ai pas besoin, sans réfléchir et vite \%. Cette réaction est très consciente, mais elle n'est pas la plus courante, avec toutes les nuances d'absence de conscience que secrète le médium: "On ne l'entend plus», traduisons: "On se laisse conditionner sans en être conscient ». Ce qu'il ne faut pas confondre avec une approbation. C'est pourquoi il est urgent, avant que nous ne soyons trop anesthésiés, de lancer un vigoureux cri d'alerte ef d'éveil auprès de l'opinion et des pouvoirs publics.

Notre passivité serait mortelle, elle équivaudrait à un lent suicide. Pire, elle nous « chosifierait» progressivement comme ces citoyens du Meilleur des Mondes de Huxley ${ }^{(7)}$, les classes alpha, bêta, gamma, delta et epsilon, dont il est étrangement troublant de sentir le futur utopique dans notre présent vécu. Notre « musique douce » fait penser un peu à leur SOMA, sorte de remède universel et de drogue à la fois, avalée aussitôt que la moindre contrariété apparaît, ou que la réalité se fait pressentir. Vite, un peu de SOMA et tout $s^{\prime}$ arrange, toute réflexion disparaît avec, j'imagine, la douleur associée à l'acte de penser. Seul, grâce au Sauvage, le poète résiste ayant encore un reste de sens critique, ce qui lui vaut d'être envoyé en exil: ce serait contraire à l'hygiène de le tolérer, car il pourrait contaminer le peuple. D'ailleurs, lui diton, en guise de consolation, il retrouvera là-bas la compagnie agréable d'autres exilés qui furent eux aussi trop éveillés à la réflexion.

\section{Fable?}

Plutôt: rappel du futur.
(7) Aldous Huxley, Brave New World, 1932, chapitre XV. 


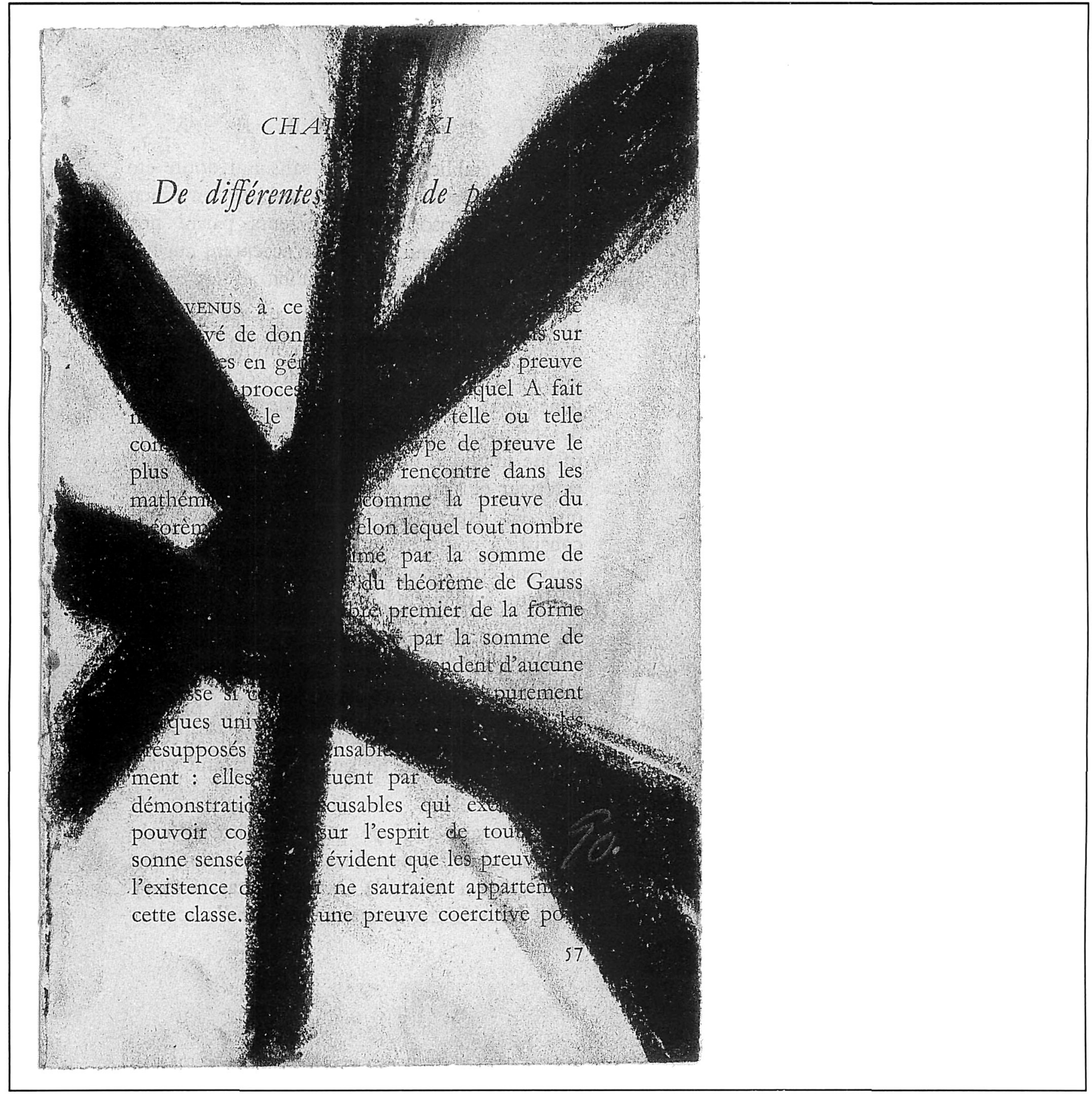

